\title{
Comparison of the Orientation of the Corneal Steep Meridian Determined by Image-Guided System and Manual Method in the Same Eye
}

This article was published in the following Dove Press journal: Clinical Ophthalmology

\author{
Tsutomu Ohashi' \\ Takashi Kojima ${ }^{2}$ \\ 'Ohashi Eye Center, Sapporo, Japan; \\ ${ }^{2}$ Department of Ophthalmology, Keio \\ University School of Medicine, Tokyo, \\ Japan
}

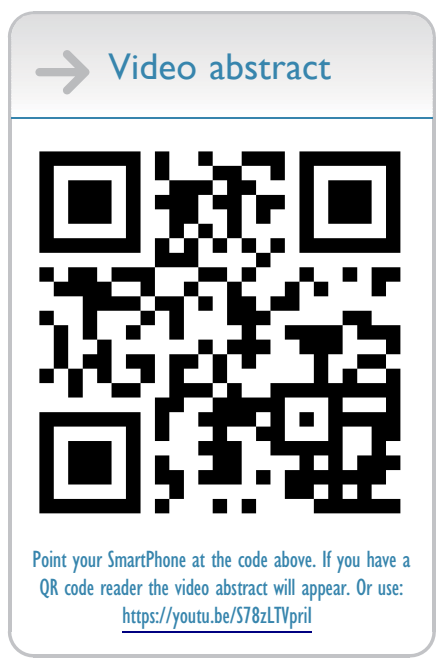

Correspondence: Tsutomu Ohashi Ohashi Eye Center, Kital-I Hondori6, Shiroishi, Sapporo 003-0027, Japan

Tel +8I- I I-864-4656

Fax +8I-II-864-2344

Email Ohashi@rainbow.ne.jp
Purpose: To evaluate the difference between the preoperative marking methods for toric intraocular lens (IOL) implantations using an image-guided system (IGS) and the manual marking method in the same eye.

Patients and Methods: In this retrospective case series, 82 patients (101 eyes) who underwent cataract surgery using both manual and IGS (VERION, Alcon Laboratories) marking were enrolled. First, preoperative reference marks were placed at 6 o'clock and 3 or 9 o'clock position under slit-lamp biomicroscope in the outpatient department using the manual method. Using the reference unit of IGS, the ocular surface data were captured and overlaid. The difference was then measured (preoperative axis misalignment). In the operating room, the orientation of the steep meridian of the manual method was determined based on this reference mark under the surgical microscope. Just before surgery, the digital degree gauge of IGS was overlaid on the ocular surface, and the difference was then measured (total axis misalignment). We calculated the intraoperative axis misalignment by subtracting preoperative axis misalignment from the total axis misalignment.

Results: Mean absolute preoperative, intraoperative, and total axis misalignment values were $3.87 \pm 3.95$ degrees, $5.46 \pm 4.42$ degrees, and $4.98 \pm 4.49$ degrees, respectively. In preoperative, intraoperative, and total misalignment, the ratios of 10 degrees or greater were 10 (14.7\%), 12 (17.6\%), and 20 (19.8\%) eyes, respectively.

Conclusion: The manual method that determines the fixed position of the toric intraocular lens (IOL) may cause large misalignment compared with the IGS, suggesting that using manual method could sometimes result in a large misalignment of toric IOL implantation.

Keywords: cataract surgery, image-guided system, steep meridian, manual method

\section{Plain Language Summary}

IGS reportedly has a small advantage compared to the manual marking method for deciding the position of steep meridian for toric lens implantation with respect to UDVA and misalignment of toric lens from previous studies. We noticed a difference between the two methods was sometimes bigger than we thought. So we decided to operate this study, to measure how difference of the misalignment between the two methods in the same eye. We find that a difference of more than 10 degrees between the two methods was sometimes found in misalignment in outpatient department and intraoperative misalignment on surgical table, indicating that the IGS advantage is larger than previously thought. It is necessary to be cautious about deciding the position of steep meridian by manual method. At a minimum the IGS has an advantage 
in deciding the steep meridian when implanting toric lenses. In this experiment, all the toric lenses were implanted according to the meridian decided by IGS. Some cases showed some extent of residual astigmatism and refractive errors, suggesting that not only lens power calculating formula error but also the postoperative change of power and axis orientation of steep meridian of cornea may affect the surgery outcome.

\section{Introduction}

Implantation of the toric intraocular lens (IOL) in the appropriate position is a key factor for accurately correcting astigmatism during cataract surgery. ${ }^{1-4}$ It has been reported that a misalignment of 3 degrees from the intended meridian results in an approximately $10 \%$ decrease in the astigmatism-correction effect, and a misalignment of 30 degrees results in a complete loss of this effect. ${ }^{5-7}$ Image-guided systems ${ }^{2,8-10}$ were developed for accurate implantation of toric IOLs. The use of these expensive devices for implanting toric lenses, in place of the manual method, remains controversial. ${ }^{2,11}$

We hypothesized that the manual method of determining the steep meridian angle introduces errors that result in misalignment of the toric IOL when compared with the IGS method. However, previously published reports did not demonstrate any large differences in effectiveness between the image-guided system and manual methods, and visual acuity outcomes from the two methods are comparable. ${ }^{2,11}$ These previous reports calculated the toric IOL misalignment from the intended meridian and compared it between the two methods. The final toric IOL misalignment and visual acuity are affected by many factors, including postoperative IOL rotation, toric IOL calculator error, and the accuracy of the preoperative examination. For this reason, it seems difficult to compare the error between the manual method and the image-guided system in two groups that comprise different patients. Therefore, we decided to conduct a study comparing the steep meridian positions calculated by the two methods, in the same eye.

Generally, when the steep meridian is determined by the manual method, the reference point is marked in the outpatient department, and the steep meridian is then determined from the reference point in the operating room. There are thus two steps in the manual method. The purpose of this study was to evaluate the axis misalignment in each stage, clarify the cause of the error, and eventually reduce the errors seen with the manual method.
Table I Preoperative and Postoperative Refractive and Visual Outcomes $(n=\mid 0 I)$

\begin{tabular}{|l|l|l|}
\hline Variables & Preoperative & Postoperative \\
\hline Age (years) & $73.7 \pm 11.3$ & - \\
Spherical equivalent (D) & $-1.43 \pm 4.29$ & $-0.54 \pm 3.00$ \\
Corneal astigmatism (D) & $-1.93 \pm 0.70$ & $-1.76 \pm 0.78$ \\
Uncorrected distance visual & $0.81 \pm 0.45$ & $0.26 \pm 0.34$ \\
acuity (log MAR) & & \\
Corrected distance visual acuity & $0.34 \pm 0.31$ & $-0.02 \pm 0.13$ \\
(log MAR) & & \\
Axial length (mm) & $24.21 \pm 1.78$ & - \\
Target refraction (D) & $-0.91 \pm 1.23$ & - \\
Manifest astigmatism (D) & $-2.34 \pm 1.31$ & $-0.73 \pm 0.45$ \\
\hline
\end{tabular}

Note: Data are presented as mean \pm standard deviation.

Abbreviations: D, diopter; log MAR, logarithm of minimum angle of resolution; SD, standard deviation.

\section{Patients and Methods}

This retrospective clinical study comprised 101 eyes of 82 cataract patients (Table 1) who underwent cataract surgery with toric IOL insertion (Acrysof IQ toric, SN6AT models, Alcon Laboratories) from October 2015 to December 2018. The inclusion criteria included patient monitoring for longer than a month after surgery, with corneal astigmatism over than 1.25 D. Eyes with a history of other ocular diseases, except for refractive error and cataracts, were excluded, as were eyes with intraoperative or postoperative complications. Of the 101 eyes, SN6AT3 IOL was implanted in 27 eyes, SN6AT4 IOL in 45 eyes, SN6AT5 IOL in 24 eyes, and SN6AT6 IOL in 5 eyes. This study was approved by the ethical review board (Chukyo Medical Ethical Board, UMINClinical Trials Registry ID: 000037098) and was conducted in accordance with the Declaration of Helsinki. All surgeries were performed at the Ohashi Eye Center in Sapporo, Japan. Written opt-out method was used as an alternative to informed consent.

\section{Visual and Refractive Outcomes}

The manifest spherical equivalent, total astigmatism, corneal astigmatism, uncorrected distant visual acuity (UDVA), corrected distant visual acuity (CDVA), and rotational error of toric IOL axis were evaluated preoperatively and 1 month after surgery. The postoperative position of toric IOL was measured using retroillumination image of the anterior segment optical coherence tomography (OCT), which can show the mark of a flat meridian of a toric lens. The difference between 
the intended axis and implanted toric IOL axis was defined as a rotational error of toric IOL.

The visual acuity was measured using a decimal visual acuity chart and converted to the logarithm of the minimum angle of resolution (log MAR) units.

\section{Toric Axis Marking Method}

The three-point method was performed as a preoperative manual steep axis marking, in line with previous reports. $^{1,12-14}$ The patient was observed under a slitlamp biomicroscope and asked to look straight ahead. First, preoperative reference marks were placed with a surgical marking pen at the 6 o'clock and either the 3 o'clock or 9 o'clock positions under a slit-lamp biomicroscope (Figure 1A). The ocular surface data were captured by the reference unit of the image-guided system (VERION, Alcon Laboratories) and overlaid with a digital degree gauge (Figure 1B). Then, the difference between 6 o'clock manual reference position and 270degree position determined using the image-guided system (IGS) was measured in the outpatient department. Using the same IGS data, the steep meridian was determined based on this reference mark on the operating table, using a surgical degree gauge (Mendez Degree Gauge, Asico) and axial marker (Figure 1C). The steep meridian of the IGS was then overlaid on the same eye just before starting surgery (Figure 1D). We compared the difference between the steep meridian made by the

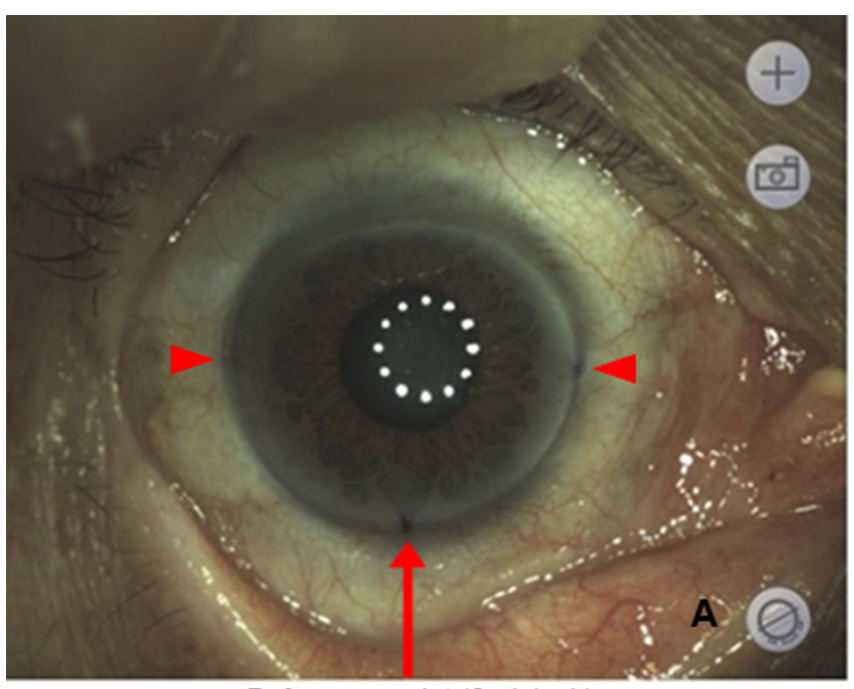

Reference point (6 o'clock)

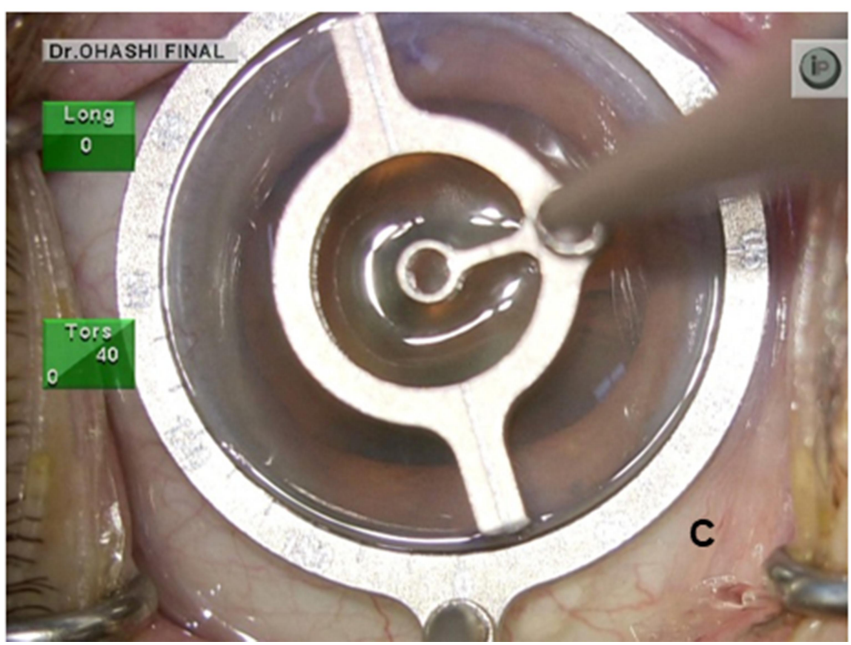

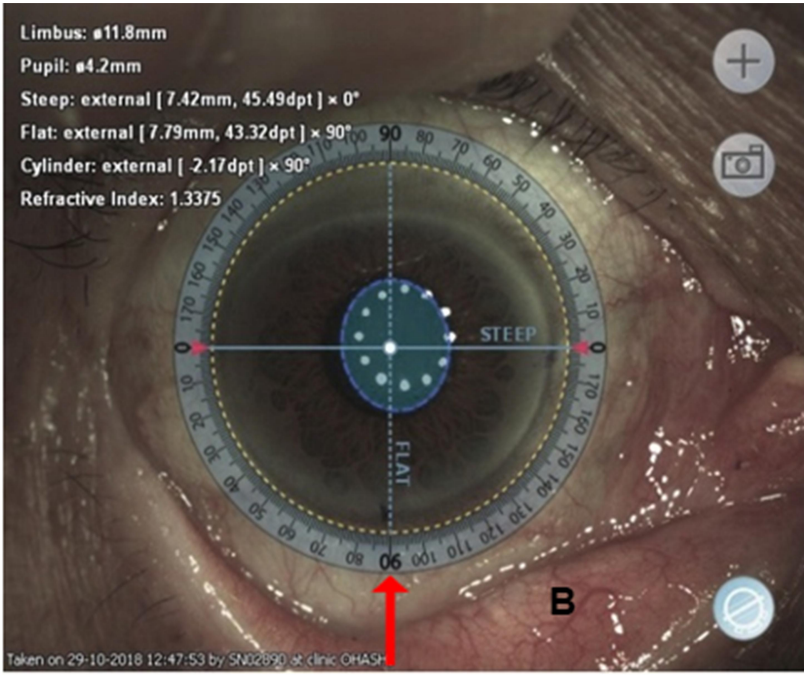

$270^{\circ}$

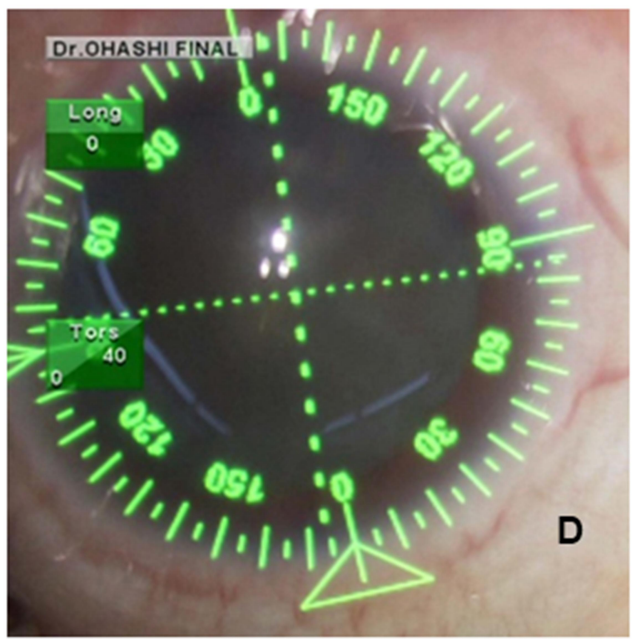

Figure I Measurement of preoperative axis misalignment and total axis misalignment. (A) Manual marking was performed using a surgical pen at three points. The red arrow shows the reference point (6 o'clock position) and the red arrow heads show the 3 and 9 o'clock positions. (B) Manual marking at the 6 o'clock position was compared with the 270-degree position (the red arrow) determined using the digital degree gauge of IGS (preoperative axis misalignment). (C) The position of the steep meridian of the manual marking was decided based on the reference mark using manual degree gauge on the operating table. (D) The position of steep meridian of IGS (green dashed line) was overlaid on the same eye just before starting surgery. The differences between them were compared (total axis misalignment). 
manual method and that made by the IGS in the surgical room (Figure 1C and D).

\section{Surgeries}

A single experienced surgeon (TO) performed phacoemulsifications and toric IOL implantations through a $2.4-\mathrm{mm}$ single plane temporal corneal incision. After removing the cataract, toric IOL was implanted according to the steep meridian, as decided by IGS.

\section{Power Calculation and Selection of the Toric IOL Models}

Ocular biometry was measured with an optical coherence biometer (IOL Master, Carl Zeiss), and the SRK/T formula was used for IOL power calculation with the A constant of 119.0 for the toric IOL. The target postoperative spherical equivalent was determined on the basis of the patient's request. The toric IOL style with respect to the toric power of the IOL and the axis location was determined using the online AcrySof toric IOL calculator. The keratometric power was measured using anterior segment OCT (CASIA SS-1000, TOMEY), and it was applied into the online AcrySof toric IOL calculator to determine the IOL power and style.

\section{Evaluation of Steep Meridian Misalignment}

The following were evaluated:

1. The difference of the reference point axes between the 6 o'clock position determined by the manual method and the 270-degree position determined by IGS (preoperative axis misalignment) was measured. When the 270-degree mark could not be observed well by a reference unit, the cases were excluded $(\mathrm{n}=33)$.

2. The differences in the steep meridian misalignments between the manual method and IGS at the operating table (total axis misalignment) were measured.

3. Intraoperative axis misalignment was calculated by subtracting the preoperative axis misalignment from the total axis misalignment. Clockwise axis misalignment was defined as positive, and counterclockwise axis misalignment as negative.

4. The correlation between the preoperative axis misalignment, intraoperative axis misalignment, and the total axis misalignment was also measured.

\section{Statistical Analysis}

Since the data did not have a Gaussian distribution, nonparametric Mann-Whitney $U$-test was used to compare the two groups. Spearman's rank correlation was applied to analyze the correlations between each step of misalignment. A p value less than 0.05 was considered statistically significant. SPSS (Version 19, IBM Corp.) was used for all statistical analyses.

Sample size calculations were carried out using the G*Power software (Heinrich Heine, Düsseldorf University, Düsseldorf, Germany) by specifying input parameters including the standard deviation, effect size, and type 1 error. $^{15}$

\section{Results}

\section{Visual and Refractive Outcomes}

The CDVA in all eyes was better than 20/20. The UDVA was better than $20 / 20$ in 28 eyes (53.8\%), and better than 18/20 in 36 eyes $(69.2 \%)$. The average manifest astigmatism decreased from $-2.34 \pm 1.31 \mathrm{D}$ to $-0.73 \pm 0.45 \mathrm{D}$ (Table 1), mean absolute rotational error of IOL axis was $3.28 \pm 2.98$ degrees $(\mathrm{N}=99)$.

\section{Evaluation of Misalignment Error}

Table 1 summarizes the outcomes following cataract surgery. Among 101 eyes, preoperative axis misalignment was measured in 68 eyes. In 33 eyes, the position of the steep meridian could not be determined because the ink marked with the surgical pen had faded, and the reference unit in the outpatient department could not be detected due to the limited resolution of the reference unit. For these reasons, preoperative axis misalignment in the outpatient department was not measured in 33 eyes. However, in the operative suite, the 6 o'clock position of the reference point was detected under the microscope in all 33 cases. In these cases, the 3, 6, and 9 o'clock positions were used as reference points during surgery. Nineteen eyes (27.9\%) showed identical position (less than 1-degree difference; Figure 2A and B), 41 eyes (60.3\%) showed less than 5 degrees of difference, but 10 eyes $(14.7 \%)$ showed a difference greater than or equal to 10 degrees (Figure $2 \mathrm{C}$ and $\mathrm{D}$ ). The mean absolute preoperative axis misalignment was 3.87 \pm 3.95 degrees (Figure 3A).

Next, the total axis misalignment was measured in 101 eyes. The absolute mean total axis misalignment was 4.98 \pm 4.49 degrees $(\mathrm{N}=101$; Figure 3B). Twenty-four eyes (23.8\%) showed an identical position (difference less than 1 degree; Figure 2A and B), 53 eyes (52.5\%) showed less than 5 degrees of difference, and 81 eyes $(80.2 \%)$ showed $1-9$ degrees of difference. However, 20 eyes (19.8\%) showed 

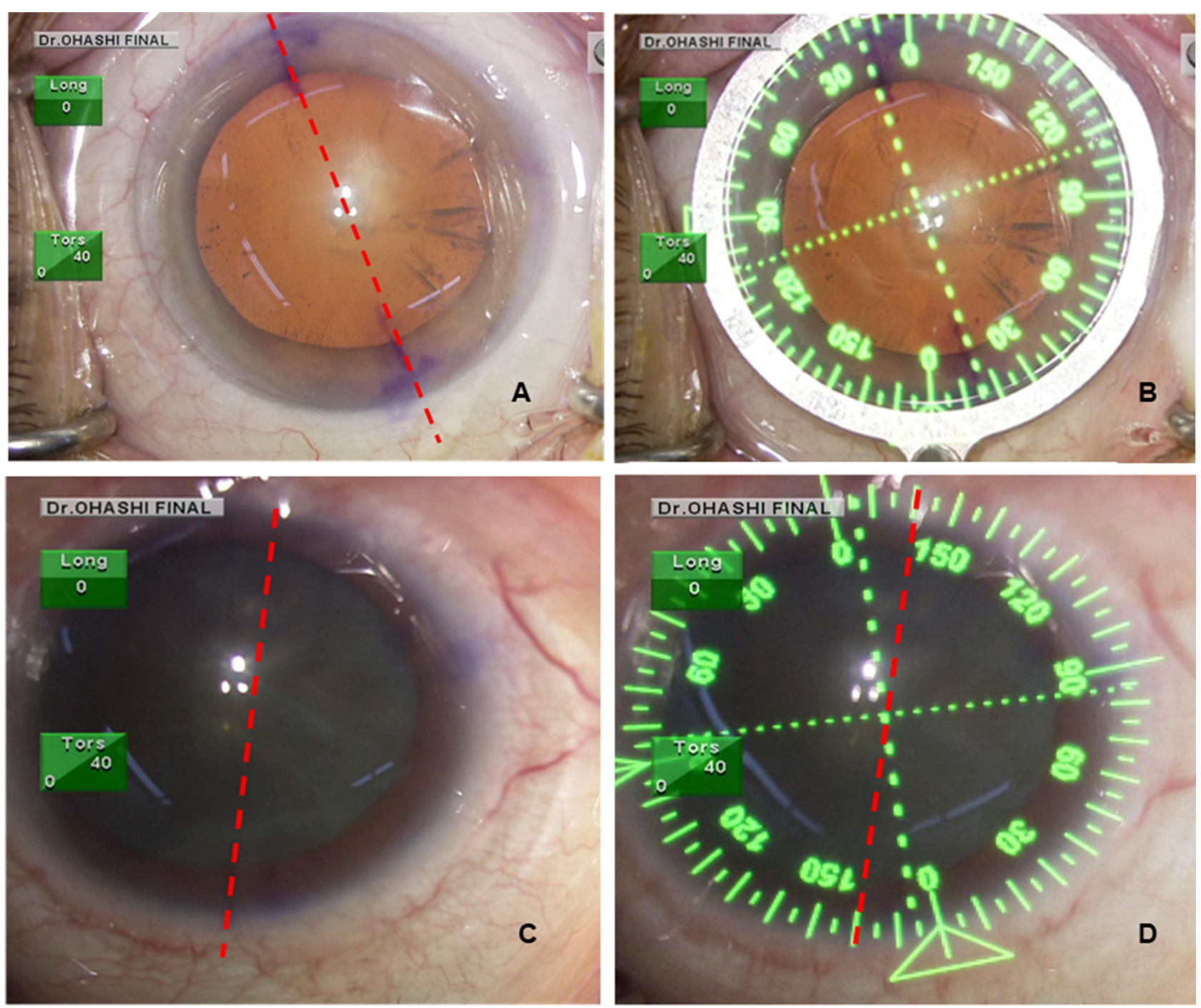

Figure 2 Representative cases with no significant or significant difference between manual method and IGS. (A and B) This case shows less than I-degree misalignment. (C and D) This case shows more than 10 degrees misalignment. The red dashed line (A, $\mathbf{C}$ and $\mathbf{D})$ shows steep meridian decided by manual method, the green dashed (B and $\mathbf{D}$ ) line show steep meridian decided by IGS.

a difference greater than or equal to a 10-degree difference (Figure 2C and D).

Next, based on the total axis misalignment and preoperative axis misalignment, the intraoperative axis misalignment (the total axis misalignment - preoperative axis misalignment) was calculated, and it was 5.46 \pm 4.42 degrees (Figure 3C).

There was no correlation between preoperative axis misalignment and total axis misalignment $(\mathrm{P}=0.13$; Figure 4A). Similarly, there was no significant correlation between the intraoperative axis misalignment and the total axis misalignment $(\mathrm{P}=0.38$; Figure $4 \mathrm{~B})$. However, there was a significant correlation between the intraoperative axis misalignment and preoperative axis misalignment $(\mathrm{R}=0.31, \mathrm{P}<0.01$; Figure $4 \mathrm{C})$.

\section{Discussion}

According to an anonymous American Society of Cataract and Refractive Surgery (ASCRS) survey conducted in $2017,{ }^{16} 60 \%$ of doctors agreed that a 5 -degree toric IOL axis misalignment is significant, and several papers have shown that a 10-degree difference can significantly affect the quality of vision. ${ }^{5,17,18}$

In this study, the toric IOL axis was adjusted using the IGS during surgery. The difference between the mean intended axis and the implanted toric IOL axis was 3.28 \pm 2.98 degrees, which indicates that the IGS can help implant the toric IOL in the correct position.

In this study, $19.8 \%$ of cases showed a total axis misalignment greater than 10 degrees (Figure 3B), suggesting that the manual method and IGS could each indicate 
A

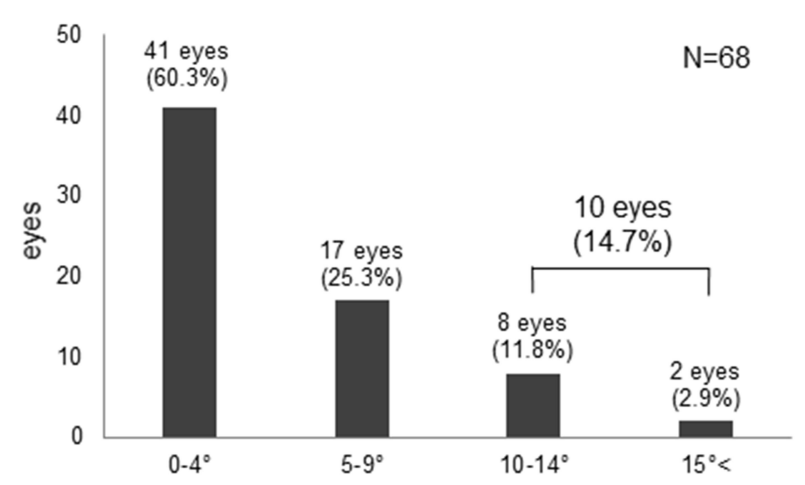

B

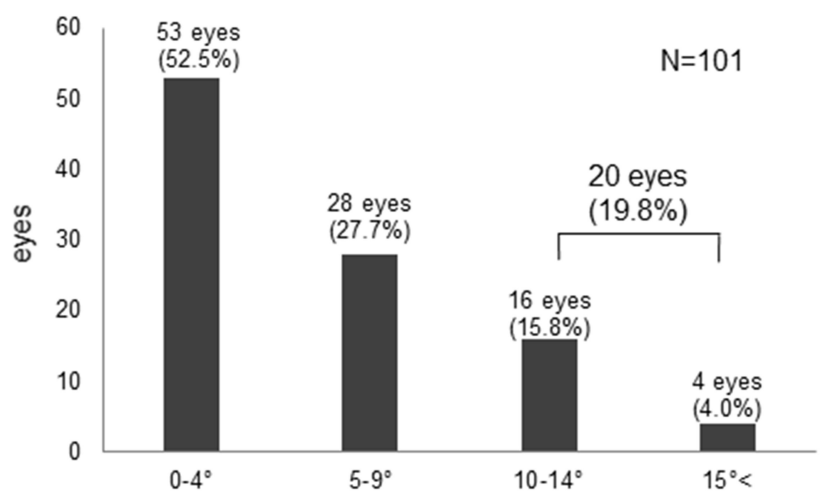

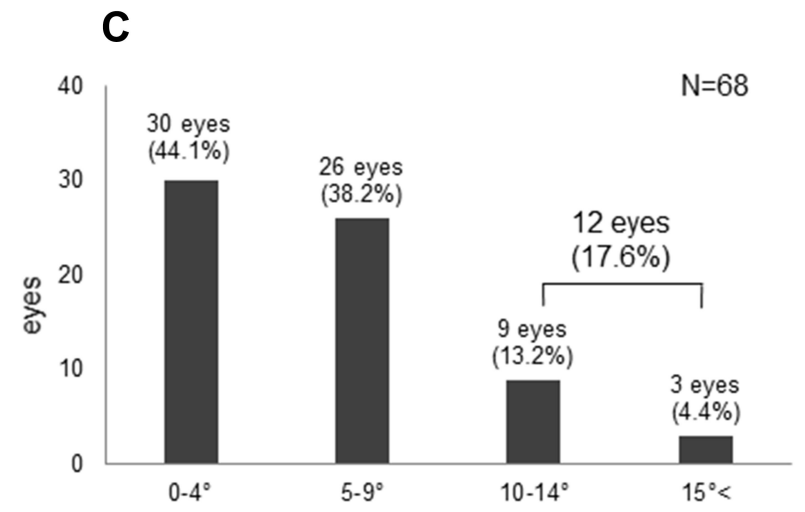

Figure 3 Distribution of axis misalignments between the manual method and IGS. (A) Preoperative axis misalignment (3.87 \pm 3.95 degrees), (B) total axis misalignment (4.98 \pm 4.49 degrees), and (C) intraoperative axis misalignment (5.46 \pm 4.42 degrees) are shown.

largely different axes on the same eye, which definitely affect postoperative refractive outcomes. The reference points (6 o'clock position in the manual method and 270degree position in the IGS) differed by more than 10 degrees in $14.7 \%$ of the cases (Figure 3A). When the position of the reference point is decided under slit-lamp biomicroscopy in the outpatient department, the Bell phenomenon as well as eye movements sometimes cause a large misalignment; this is because there is no fixation target for patients in the manual method, which makes it very difficult to manually mark the accurate 270-degree position.

In our study, $17.6 \%$ of cases showed intraoperative axis misalignment greater than 10 degrees (Figure 3C). When the position of steep meridian is decided in the operating room, it is also technically difficult to place a degree gauge on the center of the eye. This is because the size of the eyes varies between patients and because the cornea is not a perfect circle. Since one scale of the degree gauge is 10 degrees, fine adjustment is technically difficult. In addition, the ink marks are wide, and it can be difficult to determine which location is accurate.

Several previous studies have compared manual and IGS methods for toric IOL axis alignment. ${ }^{2,11,19-21}$ These papers are based on clinical comparative studies where two groups of patients were compared. In one group of eyes, the manual method was used for deciding the position of the steep meridian of the cornea. In the other group of eyes, IGS was used for deciding the position of the steep meridian of the cornea. After surgery, visual acuity, residual astigmatism, and the misalignment between the intended meridian of the cornea and the toric IOL axis mark were compared. Their results showed a small advantage for IGS compared to the manual method. Postoperative change of IOL position, measurement accuracy of corneal steep meridian, and errors of IOL power calculation formula can affect the outcome of surgeries, and therefore these factors may conceal the difference between these two methods. As Panagiotopoulou mentioned, ${ }^{22}$ IOL design, material, inadequate removal 
A

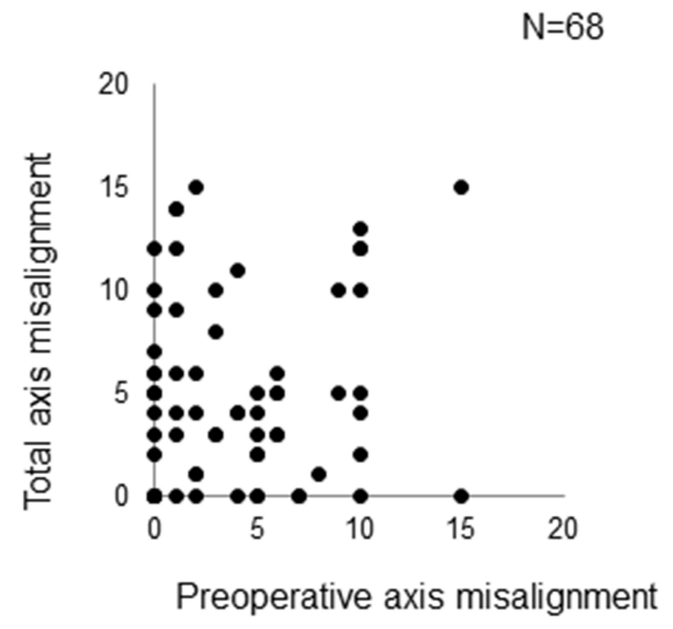

B

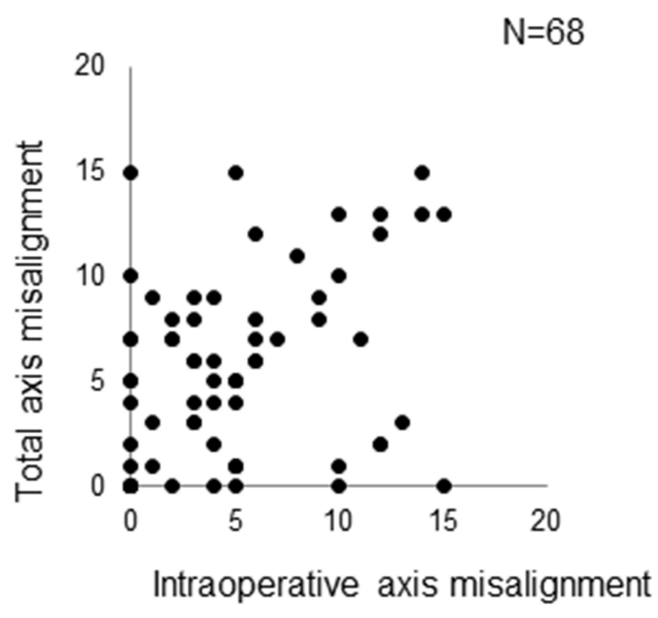

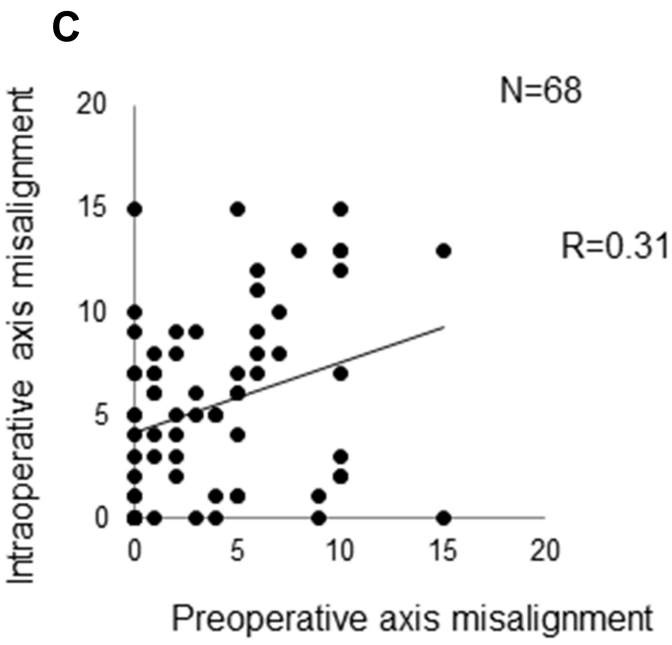

Figure 4 Correlation analyses between each misalignment. (A) No statistically significant correlation between preoperative axis misalignment and total axis misalignment of the cornea $(p=0.13)$. (B) No significant correlation between total axis misalignment and intraoperative axis misalignment $(p=0.38)$. $(\mathbf{C}) A$ significant correlation between intraoperative axis misalignment and preoperative axis misalignment $(R=0.31, p<0.01)$.

of the ocular viscosurgical devices (OVDs) from the bag postoperatively, the long $\mathrm{AL}$, the unsuitable size and centration of capsulorhexis may also affect the stability of the IOL. On the other hand, one report showed ${ }^{23}$ large difference in the horizontal axis decided by manual method and image-guided system (Calisto). They found that manual method could result in an error, which was an average 4.7 degree difference compared to image-guided system. Our results were similar to this report. However, in addition to the preoperative value, we also evaluated the intraoperative misalignment and the intended meridian misalignment between the two methods.

One of the causes of the prediction error in the postoperative astigmatism axis is the prediction error of surgical induced astigmatism (SIA). We applied $-0.1 \mathrm{D}$ for SIA (temporal incision $2.4 \mathrm{~mm}$ ) and the mean corneal astigmatism changed from $-1.93 \mathrm{D} \pm 0.7$ to $-1.76 \pm 0.78 \mathrm{D}$ after surgery (Table 1). However, axis of corneal astigmatism varied a lot more than we expected. $85 \%$ showed less than 10 degrees but $15 \%$ showed more than 10 degrees. Especially patients with small astigmatism tended to show a large change (T3 and T4 model ( $\mathrm{n}=69): 15 \%$ more than 10 degree) (T5 and T6 model ( $\mathrm{n}=28): 2 \%$ more than 10 degree) (data not shown). This may be in part due to some errors of keratometer, but further investigation is necessary. Recent techniques have made it possible to measure refractive error with an intraoperative aberrometer before implanting an IOL during surgery. Since there is a limit to the prediction of the postoperative astigmatism axis including SIA, intraoperative measurement may become important in the future. 
The IGS has two advantages. First, IGS has a fixation target within the system. We can use the same angle coordinates of another machine like a keratometer. Secondly, IGS can overlay the digital degree gauge at the operating table on the same position of the ocular surface in the outpatient department as a reference unit. In the operating room, an assistant can always double check the location of the digital degree gauge using the photos of the reference unit on the screen of the surgical pilot of IGS. If a misalignment is found, adjustment can be made to the accurate position of the intended meridian.

Initially, we expected that the greater the preoperative axis misalignment, the greater the final total axis misalignment. Similarly, we expected that if the intraoperative axis misalignment is large, the final total axis misalignment would also be large. However, there was no correlation between the preoperative axis misalignment and the total axis misalignment (Figure 4A). In addition, there was no correlation between the intraoperative axis misalignment and total axis misalignment during surgery (Figure 4B). However, there was a significant weak correlation between preoperative axis misalignment and intraoperative misalignment (Figure 4C). This may be attributable to the fact that there are common factors that affect both intraand preoperative axis misalignment. We speculated that the fixation status of the patient and the anatomical factors of the eye, such as corneal diameter, may influence the two misalignments. However, because the correlation was not so strong, another unknown factor may be involved.

Through this study, we learned that to reduce the misalignment in the manual method, a slit-lamp photo should be taken after marking to check if the 270-degree position is correct or not; if there is a misalignment, we should adjust it before we put the degree gauge on intraoperatively. Moreover, the placement of the degree gauge itself may also cause misalignment. To reduce this misalignment, it is important to place the degree gauge at the correct position. This may be achieved by instructing the patient to stare at a fixation light in the center of the microscope to decide the correct position of the degree gauge before placement. In addition, we used a Mendez gauge, which has three steps for marking. A Barrett dual axis toric marker, which has two-step marking, may also be helpful. ${ }^{24}$

This study has several limitations. First, we retrospectively studied cases of simultaneous use of manual marking and IGS shortly after introducing IGS to the clinic. The research was also conducted by a single surgeon at a single center. Randomized, large multi-center studies comparing the manual method and IGS are required to validate our findings. The IGS was used to finally adjust the position of the toric IOL. During IGS, there were cases where the intended axis and the achieved axis of a toric IOL were significantly different. It is possible that there was an error in measurement using the IGS or the IOL rotated after surgery. Further detailed research is required to elucidate why this could happen.

Ocular axis was determined based on the three markings performed using the manual method, but there is an axis registration method that was reported to be superior to the simple manual method. ${ }^{25}$ After approximately marking the horizontal axis in the spine position on the operating table, they used a video-keratoscopy to measure the axis of this reference mark in the sitting position. If there is a misalignment, they adjust this horizontal axis and begin surgery. This method may have an advantage over the manual method because they can reduce the horizontal axis misalignment. This method should be compared with the simple manual method and also with IGS in the future.

\section{Conclusion}

The meridian determined by manual markings sometimes differs greatly from that determined by IGS in the same eye, suggesting that using the manual method could sometimes result in a large misalignment of toric IOL implantation. Therefore, IGS has an advantage at least for implanting toric IOL to the intended steep meridian, compared to the manual method. Further studies are required to clarify the cause of preoperative/intraoperative axis misalignment.

\section{Abbreviations}

CDVA, corrected distant visual acuity; IGS, image-guided system; IOL, intraocular lens; log MAR, logarithm of the minimum angle of resolution; OCT, optical coherence tomography; UDVA, uncorrected distant visual acuity.

\section{Ethics Approval and Informed Consent}

This study was approved by the ethical review board (Chukyo Medical Ethical Board, UMIN-Clinical Trials Registry ID: 000037098) and was conducted in accordance with the Declaration of Helsinki. All surgeries were performed at the Ohashi Eye Center in Sapporo, 
Japan. An opt-out method was used as an alternative to informed consent.

\section{Acknowledgments}

The results of this research were presented at the Annual Meeting of American Academy of Ophthalmology in December 2019 in San Francisco in the United States.

\section{Author Contributions}

All authors made a significant contribution to the work reported, whether that is in the conception, study design, execution, acquisition of data, analysis and interpretation, or in all these areas; took part in drafting, revising or critically reviewing the article; gave final approval of the version to be published; have agreed on the journal to which the article has been submitted; and agree to be accountable for all aspects of the work.

\section{Disclosure}

Dr. Kojima declares personal fees from STAAR Surgical, Santen Pharmaceutical, Otsuka Pharmaceutical, Johnson \& Johnson, Alcon, Carl Zeiss Meditec and Echo Electricity, outside the submitted work. The authors report no other conflicts of interest in this work.

\section{References}

1. Visser N, Berendschot TT, Bauer NJ, Jurich J, Kersting O, Nuijts RM. Accuracy of toric intraocular lens implantation in cataract and refractive surgery. J Cataract Refract Surg. 2011;37(8):1394-1402. doi:10.1016/j.jcrs.2011.02.024

2. Elhofi AH, Helaly HA. Comparison between digital and manual marking for toric intraocular lenses: a randomized trial. Medicine (Baltimore). 2015;94(38):e1618. doi:10.1097/MD.0000000000001618

3. Oshika T, Inamura M, Inoue Y, et al. Incidence and outcomes of repositioning surgery to correct misalignment of toric intraocular lenses. Ophthalmology. 2018;125(1):31-35. doi:10.1016/j.ophtha.2017.07.004

4. Visser N, Bauer NJ, Nuijts RM. Toric intraocular lenses: historical overview, patient selection, IOL calculation, surgical techniques, clinical outcomes, and complications. J Cataract Refract Surg. 2013;39 (4):624-637. doi:10.1016/j.jcrs.2013.02.020

5. Langenbucher A, Viestenz A, Szentmáry N, Behrens-Baumann W, Viestenz A. Toric intraocular lenses - theory, matrix calculations, and clinical practice. $J$ Refract Surg. 2009;25(7):611-622. doi:10.3928/1081597X-20090610-07

6. Viestenz A, Seitz B, Langenbucher A. Evaluating the eye's rotational stability during standard photography: effect on determining the axial orientation of toric intraocular lenses. $J$ Cataract Refract Surg. 2005;31(3):557-561. doi:10.1016/j.jcrs.2004.07.019

7. Carey PJ, Leccisotti A, McGilligan VE, Goodall EA, Moore CB. Assessment of toric intraocular lens alignment by a refractive power/ corneal analyzer system and slitlamp observation. $J$ Cataract Refract Surg. 2010;36(2):222-229. doi:10.1016/j.jcrs.2009.08.033
8. Coleman MJ, Stark WJ, Daoud Y. A comprehensive guide to managing astigmatism in the cataract patient. Expert Rev Ophthalmol. 2014;9(6):539-544. doi:10.1586/17469899.2014.967217

9. Chaudhary G, Holladay JT, Sarayba M, Srass H. Surgical guidance and planning software for astigmatism treatment. US Patent Application 14/312,187. Filed 2014 June 23.

10. Ventura BV, Wang L, Weikert MP, Robinson SB, Koch DD. Surgical management of astigmatism with toric intraocular lenses. Arq Bras Oftalmol. 2014;77(2):125-131. doi:10.5935/0004-2749.20140032

11. Webers VSC, Bauer NJC, Visser N, Berendschot TTJM, van den Biggelaar FJHM, Nuijts RMMA. Image-guided system versus manual marking for toric intraocular lens alignment in cataract surgery. $J$ Cataract Refract Surg. 2017;43(6):781-788. doi:10.1016/j. jcrs.2017.03.041

12. Onishi H, Torii H, Watanabe K, Tsubota K, Negishi K. Comparison of clinical outcomes among 3 marking methods for toric intraocular lens implantation. Jpn $J$ Ophthalmol. 2016;60(3):142-149. doi:10.1007/s10384-016-0432-6

13. Popp N, Hirnschall N, Maedel S, Findl O. Evaluation of 4 corneal astigmatic marking methods. J Cataract Refract Surg. 2012;38 (12):2094-2099. doi:10.1016/j.jcrs.2012.07.039

14. Kim MH, Chung TY, Chung ES. Long-term efficacy and rotational stability of AcrySof toric intraocular lens implantation in cataract surgery. Korean J Ophthalmol. 2010;24(4):207-212. doi:10.3341/ kjo.2010.24.4.207

15. Faul F, Erdfelder E, Lang AG, Buchner A. G*Power 3: a flexible statistical power analysis program for the social, behavioral, and biomedical sciences. Behav Res Methods. 2007;39(2):175-191. doi:10.3758/BF03193146

16. Eyeworld Supplements. ASCRS clinical survey. Available from: http://supplements.eyeworld.org/eyeworld-supplements/2017-ascrsclinical-survey-supplement-dl-final. Accessed March 23, 2020.

17. Novis C. Astigmatism and toric intraocular lenses. Curr Opin Ophthalmol. 2000;11(1):47-50. doi:10.1097/00055735-20000200000007

18. Ma JJ, Tseng SS. Simple method for accurate alignment in toric phakic and aphakic intraocular lens implantation. $J$ Cataract Refract Surg. 2008;34(10):1631-1636. doi:10.1016/j.jcrs.2008.04.041

19. Nochioka K, Hayashi R, Natsume K, Ijuin N, Mtsuura T, Ogata N. Axis registration method compared with 3 and 9 o'clock marking method for toric intraocular lens implantation. Jpn J Cataract Refract Surg. 2014;28:86-89.

20. Ichikawa K, Oshika S, Tomiyasu S, et al. Visual outcome of different axis marking methods for toric intraocular lens implantation at multiple institutions. Jpn J Cataract Refract Surg. 2014;28:294-298.

21. Zhou F, Jiang W, Lin Z, et al. Comparative meta-analysis of toric intraocular lens alignment accuracy in cataract patients: image-guided system versus manual marking. $J$ Cataract Refract Surg. 2019;45(9):1340-1345. doi:10.1016/j.jcrs.2019.03.030

22. Panagiotopoulou EK, Ntonti P, Gkika M, et al. Image-guided lens extraction surgery: a systematic review. Int J Ophthalmol. 2019;12 (1):135-151.

23. Raucau M, El Chehab H, Agard E, Lagenaite C, Dot C. Toric lens implantation in cataract surgery: automated versus manual horizontal axis marking, analysis of 50 cases. J Fr Ophthalmol. 2018;41(1):e1e9. doi:10.1016/j.jfo.2017.11.002

24. Lipsky L, Barrett G. Comparison of toric intraocular lens alignment error with different toric markers. J Cataract Refract Surg. 2019;45 (11):1597-1601. doi:10.1016/j.jcrs.2019.06.013

25. Suzuki A, Maeda N, Watanabe H, Kiritoshi A, Shimomura Y, Tano Y. Using a reference point and videokeratography for intraoperative identification of astigmatism axis. J Cataract Refract Surg. 1997;23 (10):1491-1495. doi:10.1016/S0886-3350(97)80019-3 


\section{Publish your work in this journal}

Clinical Ophthalmology is an international, peer-reviewed journal covering all subspecialties within ophthalmology. Key topics include: Optometry; Visual science; Pharmacology and drug therapy in eye diseases; Basic Sciences; Primary and Secondary eye care; Patient Safety and Quality of Care Improvements. This journal is indexed on PubMed
Central and CAS, and is the official journal of The Society of Clinical Ophthalmology (SCO). The manuscript management system is completely online and includes a very quick and fair peer-review system, which is all easy to use. Visit http://www.dovepress.com testimonials.php to read real quotes from published authors. 